\title{
Algoritmo de caminho mínimo para grafos coloridos aplicado a redes de transporte multimodal
}

\author{
Juliana V. Shirabayashi \\ Universidade Federal do Paraná - Campus avançado de Jandaia do Sul \\ Rua Dr. João Maximiano, 426 \\ Jandaia do Sul, PR \\ E-mail: juliana.verga@ufpr.br \\ Akebo Yamakami \\ Departamento de Sistemas e Energia - FEEC - UNICAMP \\ Av. Albert Einstein, 400 \\ 13083-852, Campinas, SP \\ E-mail: akebo@dt.fee.unicamp.br \\ Ricardo C. Silva \\ Departamento de Estatística e Matemática Aplicada - UFC \\ Rua Campus do Pici, Bloco 910 \\ 60440554, Fortaleza, CE \\ E-mail: rcoelhos@gmail.com \\ Wesley Vagner I. Shirabayashi \\ Departamento de Matemática - UEM \\ Av. Colombo, 5790 \\ 87020-900, Maringá, PR \\ E-mail: wvishirabayashi@uem.br
}

Resumo: Este trabalho apresenta um algoritmo para o problema de caminho mínimo em grafos coloridos baseado no algoritmo clássico de Ford-Moore-Bellman e aplicado a redes de transporte multimodal, onde cada modo de transporte considerado é representado por uma cor, podendo ter vários arcos entre dois nós do grafo. Durante o procedimento de resolução, o método detecta se houve mudança de modo e um custo referente a esta mudança é acrescentado no caminho, ao final temos um caminho mínimo multimodal com informação de qual modo foi utilizado para percorrer cada arco do caminho. Testes computacionais foram feitos a fim de comprovar a viabiliade do algoritmo proposto.

Palavras-chave: caminho minimo, grafos coloridos, redes de transporte multimodal.

\section{Apresentação do problema}

Um dos fatores que afetam a qualidade de vida em uma área metropolitana é o sistema de transporte local. Redes de transporte urbanas são cada vez mais caracterizadas por congestionamentos e seu impacto correspondente na acessibilidade individual, na poluição do ar e no desenvolvimento de atividades econômicas urbanas [6]. Um fato que ocorre na maioria das cidades é que o local de trabalho das pessoas, muitas vezes, é longe da casa, o que faz com que utilizem mais de um meio de transporte para se deslocar de sua residência até o local de trabalho. 
Sistemas de transportes são considerados multimodais pois geralmente contêm vários modos de transportes, tais como: ônibus, metrô, trem, dentre outros. Dessa forma, os usuários podem utilizar vários modos de transporte em uma viagem e geralmente não estão dispostos a trocar muitas vezes de meio de transporte. Vários parâmetros são considerados pelos usuários ao usar vários meios de transporte, como por exemplo: o número de mudanças de modo, o percurso da viagem, tempo de percurso, que devem ser os mais curtos possíveis [6]. Isto nos leva ao problema de caminho mínimo, para o qual existem diversos algoritmos. Porém, considerando uma rede multimodal e seus parâmetros associados, tais algoritmos se tornam insuficientes para orientar os usuários corretamente. Várias extensões tem sido propostas para suprir tais deficiências $[3,5,7]$.

Quanto à formulação, a teoria de grafos é comumente usada neste caso. Ela fornece uma modelagem consistente do problema de redes de transporte multimodal e de muitos outros problemas, facilitando a implementação de algoritmos que auxiliam na obtenção da sua solução [1]. A coloração em grafos é um problema de otimização combinatória bastante estudado e que possui variantes: coloração em nós, coloração em arestas, coloração em faces, dentre outras. Muitos problemas práticos podem ser modelados através da coloração em grafos, dentre eles: gerência e alocação de recursos, redes de telecomunicação, redes de transporte, dentre outros.

Este trabalho apresenta um método baseado no algoritmo clássico de caminho mínimo de Ford-Moore-Bellman [2] e tem como objetivo encontrar os caminhos mínimos multimodais em grafos coloridos com custos crisp, isto é custos cujos valores escalares. O termo crisp é usado para diferenciar, por exemplo, custos fuzzy de custos clássicos (crisp).

\section{Formulação matemática do problema}

Um problema bem conhecido em teoria de grafos envolvendo cores é o problema de coloração em grafos. Este problema lida com a atribuição de cores nos elementos de um grafo de acordo com determinadas restrições. Coloração em nós é uma variante deste problema, neste caso o objetivo é colorir os nós de um grafo usando o menor número de cores tal que dois nós adjacentes tenham cores diferentes. Analogamente, o problema de coloração em arcos trata da atribuição de cores nos arcos de um grafo usando o menor número de cores tal que dois arcos consecutivos tenham cores diferentes.

A coloração em grafos utilizada neste trabalho é significantemente diferente dos tipos de coloração citados acima. A coloração nos arcos não está relacionada com a atribuição de cores aos elementos do grafo de acordo com determinadas restrições, mas neste caso as cores representam os diferentes modos de transportes em uma rede e o objetivo é encontrar uma estrutura ótima da rede através de caminhos mínimos. Na literatura, encontramos um trabalho [8] que trata o problema de redes de transporte multimodal usando coloração em grafos e baseia-se no algoritmo clássico de Dijkstra [4].

Seja $G=(N, A, L)$ um multigrafo direcionado e colorido, consistindo de um conjunto de nós $(N)$, um conjunto de cores (ou rótulos) $L$ e um conjunto de $\operatorname{arcos} \operatorname{rotulados}(i, j, l)$ os quais são triplas em $N \times N \times L$, com $i, j \in N, l \in L$. Cada cor (rótulo), $l \in L$, representa um modo de transporte, dessa forma $(i, j, l)$ representa o arco ligando o nó $i$ ao nó $j$ com modo de transporte $l$.

Neste contexto, para redes de transporte monomodal pode haver apenas um arco entre cada par de nós do grafo, já para redes de transporte multimodal, pode haver mais de um arco entre dois nós do grafo, mas não necessariamente entre todos os pares de nós. Além das cores (rótulos), cada arco tem um custo, por exemplo, $c_{i j l}$ representa o custo do arco $(i, j)$ usando o modo $l$.

O problema de caminho mínimo em redes de transporte multimodal pode ser formulado da seguinte forma: 


$$
\begin{aligned}
& \min z=\sum_{(i, j, l) \in A} c_{i j l} x_{i j l} \\
& \text { s.a } \sum_{j:(i, j, l) \in A} x_{i j l}-\sum_{j:(j, i, l) \in A} x_{j i l}=\left\{\begin{array}{cc}
1, & i=o \\
0, & i \neq o, d \\
-1, & i=d
\end{array}\right. \\
& x_{i j l}=\{0,1\}, \quad \forall(i, j, l) \in A, \quad \forall l \in L .
\end{aligned}
$$

onde:

- o: nó origem;

- $d$ : nó destino;

- $x_{i j l}$ : variável de decisão.

Quanto às restrições, a primeira restrição garante que o grafo esteja balanceado, ou seja, em uma solução viável a quantidade de arcos entrando em determinado nó será igual à quandidade de arcos saindo de tal nó, a segunda restrição garante a não negatividade da variável de decisão bem como os valores que a mesma pode assumir: 1 se o arco $(i, j, l)$ pertence ao caminho ou 0 se o arco não pertence ao caminho. A função objetivo minimiza a soma dos custos dos arcos que compõem o caminho mínimo.

\section{Algoritmo proposto}

O algoritmo proposto é uma generalização do algoritmo clássico de Ford-Moore-Bellman [2] para grafos coloridos cuja aplicação será feita em redes de transporte multimodal. Tal algoritmo é iterativo, possuindo como critério de parada o número de iterações ou a não alteração dos custos encontrados na iteração anterior com relação à iteração atual.

Na generalização feita, cada nó terá um conjunto de etiquetas et $q\left(j, l_{j}\right.$, ant, rot, cust) onde:

- $j$ : nó em análise;

- $l_{j}$ : número da etiqueta do nó $j$;

- ant: guarda o nó $i$ antecedente do nó $j$ pelo caminho considerado;

- rot: guarda o modo (ou cor) utilizado para chegar de $i$ até $j$;

- cust: guarda o custo acumulado da origem até o nó (incluindo o custo de mudança de modo, quando houver).

\section{Notações para o algoritmo}

- $N$ : número de nós do grafo;

- $\Gamma_{j}^{-1}$ : conjunto dos nós predecessores do nó $j$;

- it: contador de iterações;

- $d_{i j l}$ : custo do arco $(i, j)$ pelo modo $l$;

- Nmodos: números de modos de transporte considerados;

- $\epsilon$ : custo de mudança de modo, que, neste caso é um número fixo.

A seguir temos os passos do algoritmo. 
- Passo 1: Inicialização:

- ant $=[]$.

$-\operatorname{rot}=[]$.

- $\operatorname{cust}^{0}($ origem $)=0$.

- $\operatorname{cust}^{0}(j)=\infty$ se $j \neq$ origem.

$-i t \leftarrow 1$.

- Passo 2:

Para todo $j \in N$ faça:

Para todo $i \in \Gamma^{-1}(j)$ faça:

Para todo $l_{j}$ faça:

Para todo $l \in N$ modos faça:

Se $\operatorname{rot}(j) \neq \operatorname{rot}(i)$

$\operatorname{cust}^{i t}(j)=\operatorname{cust}^{i t-1}(j)+d_{i j l}+\epsilon$

Senão

$\operatorname{cust}^{i t}(j)=\operatorname{cust}^{i t-1}(j)+d_{i j l}$

$\operatorname{ant}(j)=i$

$\operatorname{rot}(j)=l$

Fim Se

Fim $l$

Fim $l_{j}$

Fim $i$

$\operatorname{Fim} j$

- Passo 3: Critério de Parada:

1. Se $i t=i t+1 \geq$ número de arcos, ou se cust $t^{i t}=c u s t^{i t-1}, \forall j \in N$, vá para o Passo 4

2. Senão, vá para o Passo 2.

- Passo 4: Recompor os caminhos a partir das etiquetas construídas no Passo 2.

O número de etiquetas por nó tende a aumentar rapidamente à medida em que se aumenta o número de arcos e modos do problema a ser resolvido. Por exemplo, considerando que há o mesmo número de modos entre cada par de nós, em uma solução viável (caminho mínimo), o número de etiquetas é dado por: $m^{a}$, onde $m$ é o número de modos de transporte e $a$ é o número de arcos entre a origem e o destino considerado.

O algoritmo clássico de Ford-Moore-Bellman, examina todos os nós até que não seja possível melhorias, dessa forma, aceita arcos com custos negativos. Como o algoritmo proposto é baseado no algoritmo de Ford-Moore-Bellman, a estrutura é mantida.

\section{Teste computacional}

Neste exemplo, a rede multimodal é constituída de 17 nós e 52 arcos, contendo três modos de transporte. Os nós 1 e 6 são nós origem e o nó 17 é nó destino. Os arcos de cor preta (linhas) são referentes ao modo 1, os arcos de cor azul (tracejados) são referentes ao modo 2 e os arcos de cor vermelha (pontilhados) são referentes ao modo 3.

Os arcos, custos e os modos de transporte estão definidos na Tabela 1. 


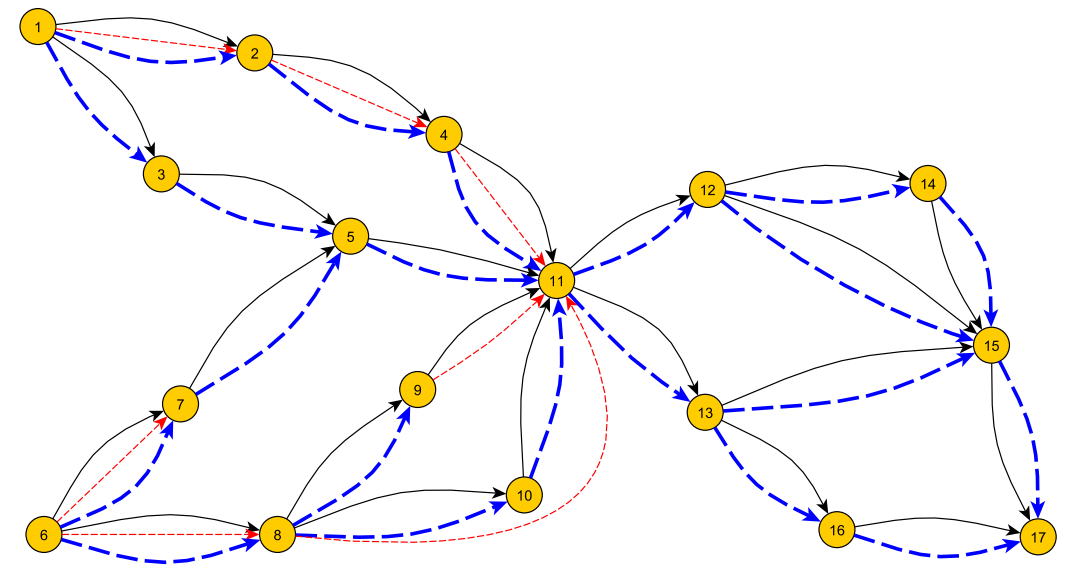

Figura 1: Rede com três modos de transporte entre alguns nós

\begin{tabular}{|c|c|c|c|c|c|c|c|}
\hline Arco & Origem $\rightarrow$ Destino & Custos & Modos & Arco & Origem $\rightarrow$ Destino & Custos & Modos \\
\hline 1 & $1 \rightarrow 2$ & 4 & 1 & 27 & $8 \rightarrow 10$ & 5 & 2 \\
2 & $1 \rightarrow 2$ & 7 & 2 & 28 & $8 \rightarrow 11$ & 13 & 3 \\
3 & $1 \rightarrow 2$ & 3 & 3 & 29 & $9 \rightarrow 5$ & 4 & 1 \\
4 & $1 \rightarrow 3$ & 2 & 1 & 30 & $9 \rightarrow 5$ & 1 & 2 \\
5 & $1 \rightarrow 3$ & 7 & 2 & 31 & $9 \rightarrow 11$ & 5 & 1 \\
6 & $2 \rightarrow 4$ & 3 & 1 & 32 & $9 \rightarrow 11$ & 2 & 3 \\
7 & $2 \rightarrow 4$ & 4 & 2 & 33 & $10 \rightarrow 11$ & 3 & 1 \\
8 & $2 \rightarrow 4$ & 7 & 3 & 34 & $10 \rightarrow 11$ & 6 & 2 \\
9 & $3 \rightarrow 5$ & 5 & 1 & 35 & $11 \rightarrow 12$ & 12 & 1 \\
10 & $3 \rightarrow 5$ & 3 & 2 & 36 & $11 \rightarrow 12$ & 2 & 2 \\
11 & $4 \rightarrow 11$ & 7 & 1 & 37 & $11 \rightarrow 13$ & 11 & 1 \\
12 & $4 \rightarrow 11$ & 6 & 2 & 38 & $11 \rightarrow 13$ & 4 & 2 \\
13 & $4 \rightarrow 11$ & 5 & 3 & 39 & $12 \rightarrow 14$ & 4 & 1 \\
14 & $5 \rightarrow 11$ & 6 & 1 & 40 & $12 \rightarrow 14$ & 5 & 2 \\
15 & $5 \rightarrow 11$ & 5 & 2 & 41 & $12 \rightarrow 15$ & 10 & 1 \\
16 & $6 \rightarrow 7$ & 2 & 1 & 42 & $12 \rightarrow 15$ & 12 & 2 \\
17 & $6 \rightarrow 7$ & 7 & 2 \\
18 & $6 \rightarrow 7$ & 2 & 3 \\
19 & $6 \rightarrow 8$ & 4 & 1 & 43 & $13 \rightarrow 15$ & 5 & 1 \\
20 & $6 \rightarrow 8$ & 5 & 2 & 45 & $13 \rightarrow 15$ & 7 & 2 \\
21 & $6 \rightarrow 8$ & 2 & 3 & 46 & $13 \rightarrow 16$ & 8 & 1 \\
22 & $7 \rightarrow 5$ & 8 & 1 & 48 & $14 \rightarrow 16$ & 9 & 2 \\
23 & $7 \rightarrow 5$ & 3 & 2 & 49 & $15 \rightarrow 17$ & 5 & 1 \\
24 & $8 \rightarrow 9$ & 3 & 1 & 50 & $15 \rightarrow 17$ & 3 & 2 \\
25 & $8 \rightarrow 9$ & 4 & 2 & 51 & $16 \rightarrow 17$ & 3 & 1 \\
26 & $8 \rightarrow 10$ & 3 & 1 & 52 & $16 \rightarrow 17$ & 2 & 2 \\
\hline
\end{tabular}

Tabela 1: Dados da Rede Multimodal da Figura 1

As Tabelas 2 e 3 apresentam os resultados obtidos através do algoritmo proposto para diferentes valores do custo de mudança de modo considerando os nós 1 e 6 como nós origem. O número em cima da seta em cada arco indica o modo de transporte usado para percorre-lo. 


\begin{tabular}{|c|c|c|c|}
\hline Custo de mudança & Caminho mínimo & Custo do caminho \\
\hline 0 & $1 \longrightarrow^{1} 3 \longrightarrow^{2} 5 \longrightarrow^{2} 11 \longrightarrow^{2} 13 \longrightarrow^{1} 15 \longrightarrow^{2} 17$ & 22 \\
0,5 & $1 \longrightarrow^{1} 3 \longrightarrow^{2} 5 \longrightarrow^{2} 11 \longrightarrow^{2} 13 \longrightarrow^{1} 15 \longrightarrow^{2} 17$ & 23,5 \\
2 & $1 \longrightarrow^{1} 3 \longrightarrow^{2} 5 \longrightarrow^{2} 11 \longrightarrow^{2} 13 \longrightarrow^{2} 15 \longrightarrow^{2} 17$ & 26 \\
4 & $1 \longrightarrow^{1} 3 \longrightarrow^{1} 5 \longrightarrow^{1} 11 \longrightarrow^{2} 13 \longrightarrow^{2} 15 \longrightarrow^{2} 17$ & 31 \\
8 & $1 \longrightarrow^{1} 3 \longrightarrow^{1} 5 \longrightarrow^{1} 11 \longrightarrow^{1} 13 \longrightarrow^{1} 15 \longrightarrow^{1} 17$ & 34 \\
\hline
\end{tabular}

Tabela 2: Resultados obtidos através do algoritmo proposto para o nó origem 1

\begin{tabular}{|c|c|c|}
\hline Custo de mudança & Caminho mínimo & Custo do caminho \\
\hline 0 & $6 \longrightarrow^{3} 8 \longrightarrow^{1} 9 \longrightarrow^{3} 11 \longrightarrow^{2} 13 \longrightarrow^{1} 15 \longrightarrow^{2} 17$ & 19 \\
0,5 & $6 \longrightarrow^{3} 8 \longrightarrow^{1} 9 \longrightarrow^{3} 11 \longrightarrow^{2} 13 \longrightarrow^{1} 15 \longrightarrow^{2} 17$ & 21,5 \\
2 & $6 \longrightarrow^{3} 8 \longrightarrow^{1} 10 \longrightarrow^{1} 11 \longrightarrow^{2} 13 \longrightarrow^{2} 15 \longrightarrow^{2} 17$ & 26 \\
4 & $6 \longrightarrow^{3} 8 \longrightarrow^{1} 10 \longrightarrow^{1} 11 \longrightarrow^{2} 13 \longrightarrow^{2} 15 \longrightarrow^{2} 17$ & 30 \\
8 & $6 \longrightarrow^{3} 8 \longrightarrow^{3} 11 \longrightarrow^{2} 13 \longrightarrow^{2} 15 \longrightarrow^{2} 17$ & 37 \\
\hline
\end{tabular}

Tabela 3: Resultados obtidos através do algoritmo proposto para o nó origem 6

Neste exemplo, fizemos testes para outros valores do custo de mudança de modo e observamos que para valores maiores ou iguais a 8, o caminho mínimo encontrado é sempre o mesmo.

\section{Conclusões}

Problemas de redes de transporte têm sido extensamente estudados e aplicados nas soluções de problemas reais. Tratar do problema de redes de transportes multimodais, monomodais e problemas correlatos é cada vez mais importante e necessário para buscar soluções, melhorar o planejamento, e, permitir uma viagem mais tranquila tanto para os usuários de transportes públicos bem como de veículos particulares.

O método apresentado tratou o problema de caminho mínimo em grafos coloridos com aplicação em redes de transporte multimodal. Ao considerar diferentes valores para o custo de mudança de modo, diferentes soluções foram encontradas, fato que ocorre em problemas reais, já que os usuários, em geral, não estão dispostos a realizar mudança de modo, mas fazem tal mudança quando não tem outra opção. Vale lembrar que este método é inovativo, pois na literatura encontramos um trabalho que propõe um algoritmo semelhante, porém baseado no algoritmo clássico de Dijkstra.

Testes em instâncias maiores estão sendo feitos a fim de comprovar a eficiência e acurácia do método, que mostrou-se eficiente no exemplo aqui apresentado.

\section{Referências}

[1] T. L. Ahuja, R. K. Magnanti, "Network Flows", Prentice Hall, Philadelphia, PA, USA, 1993.

[2] R. E. Bellman. On a routing problem. Quarterly Applied Mathematics, 16(1958), 8790.

[3] M. Bieli, A. Boumakoul, H. Mouncif, Object modeling and path computation for multimodal travel systems. Eur. J. Oper. Res., 175(2006), 1705-1730. 
[4] E. W. Dijkstra, A note on two problems in conexion with graphs. Numerische Mathematik, 1(1959), 269-271.

[5] A. Keshtiarast, A. A. Alesheikh, A. Kheirbadi, Best route finding based on cost in multimodal network with care of networks constraints, Map Asia, India, Ref no. $66(2006)$.

[6] A. Lozano, G. Storchi, Shortest viable path algorithm in multimodal networks. Transportation Research, 35(2001), 225-241.

[7] H. J. Miller, J. D. Storm, M. Bowen, GIS design for multimodal networks analysis em "Annual Conference and Exposition Proceedings of GIS/LIS", pp. 750-759, Nashville, EUA, 1995.

[8] F. E. L. Viedma, Coloured-edge graph approach for the modelling of multimodal networks, Tese de Doutorado, Auckland University of Technology, 2011. 